\title{
Continuous Real-Time in vivo Measurement of Cerebral Nitric Oxide Supports Theoretical Predictions of an Irreversible Switching in Cerebral ROS after Sufficient Exposure to External Toxins
}

\author{
Niall J. Finnerty ${ }^{\mathrm{a}, *}$, Saidhbhe L. O'Riordan ${ }^{\mathrm{a}}$, John P. Lowry ${ }^{\mathrm{a}}$, Mathieu Cloutier ${ }^{\mathrm{b}}$ and Peter Wellstead \\ ${ }^{a}$ Sensors Development Unit and Neurochemistry Research Unit, BioAnalytics Laboratory, \\ Department of Chemistry, National University of Ireland Maynooth, Co. Kildare, Ireland \\ ${ }^{\mathrm{b}}$ Institute GERAD and University of Montreal, Montreal, Canada \\ ${ }^{\mathrm{c}}$ Hamilton Institute, National University of Ireland Maynooth, Co. Kildare, Ireland
}

\begin{abstract}
.
Background: Mathematical models of the interactions between alphasynuclein $(\alpha S)$ and reactive oxygen species (ROS) predict a systematic and irreversible switching to damagingly high levels of ROS after sufficient exposure to risk factors associated with Parkinson's disease (PD).

Objectives: We tested this prediction by continuously monitoring real-time changes in neurochemical levels over periods of several days in animals exposed to a toxin known to cause Parkinsonian symptoms.

Methods: Nitric oxide (NO) sensors were implanted in the brains of freely moving rats and the NO levels continuously recorded while the animals were exposed to paraquat (PQ) injections of various amounts and frequencies.

Results: Long-term, real-time measurement of NO in a cohort of animals showed systematic switching in levels when PQ injections of sufficient size and frequency were administered. The experimental observations of changes in NO imply a corresponding switching in endogenous ROS levels and support theoretical predictions of an irreversible change to damagingly high levels of endogenous ROS when PD risks are sufficiently large.

Conclusions: Our current results only consider one form of PD risk, however, we are sufficiently confident in them to conclude that: (i) continuous long-term measurement of neurochemical dynamics provide a novel way to measure the temporal change and system dynamics which determine Parkinsonian damage, and (ii) the bistable feedback switching predicted by mathematical modelling seems to exist and that a deeper analysis of its characteristics would provide a way of understanding the pathogenic mechanisms that initiate Parkinsonian cell damage.
\end{abstract}

Keywords: Parkinson's disease, reactive oxygen species, nitric oxide, experimental models, mathematical model

\section{INTRODUCTION}

${ }^{*}$ Correspondence to: Niall Finnerty, PhD., Research Fellow, BioAnalytics Laboratory, Chemistry Department, NUI Maynooth, Ireland. Tel.: +353 01708 6477; E-mail: Niall.finnerty@ nuim.ie.
Along with other neurodegenerative conditions, Parkinson's disease (PD) poses a growing threat to our aging societies. The main risk factor for PD is advanced age [1], while other issues such as exposure 
to agricultural and industrial toxins, head trauma and genetic predisposition also create an increased vulnerability. While the sources of risk are known, we do not understand the detail of how PD risk factors cause the initiation of self-sustaining Parkinsonian damage within brains that leads to pathogenesis [2]. In particular, while there are qualitative descriptions of the development of Parkinsonian damage as a 'vicious cycle' of neurochemical interactions [3], there are no mathematical models for how the vicious cycle might work, and no in vivo means of continuously monitoring the temporal changes in neurochemicals involved in the cycle.

In recent years however, two developments (one theoretical and one experimental) have occurred which address these limitations. In the theoretical development, mathematical modelling has been used to simulate and conduct theoretical analysis of neurochemical bi-stability in cells under stress from PD risk factors $[4,5]$. Computational and theoretical analysis of the models predicts that vulnerable neurons have a switching property that (when under sufficiently severe pressure from PD risk factors) causes them to irreversibly change to a Parkinsonian state. In particular, mathematical modelling of interactions between reactive oxygen species (ROS) and alphasynuclein $(\alpha S)$ predicts that the key functional mechanism in the 'vicious cycle' associated with Parkinsonian damage is a bistable feedback switch that resembles known biological motifs [6].

The experimental development is a sensing technology for continuously monitoring the real-time variations of specific neurochemicals in the brains of freely moving animals. Using Long Term In Vivo Electrochemistry (LIVE) it is now possible to measure with great specificity and sensitivity the relative concentrations of neurochemicals [7-9]. Animals fitted with LIVE sensors can be used to study in real-time the temporal changes in neurochemical levels that occur after exposure to disease risk factors. For example, LIVE has been used to study neurochemical changes in an animal model of schizophrenia [10]. In the current article we extend the use of LIVE to measure instantaneous neurochemical changes in animals exposed to a particular form of PD risk factor, and use the experimental results to assess theoretical predictions of a systematic neurochemical switching in the pathogenesis of Parkinsonian damage within a cerebral compartment. Theoretically, the compartment can consist of a region within a neuron, a single neuron, a group of neurons or an entire brain area. In the context of this paper, the cerebral compartment is the region immediately surrounding the LIVE sensors.
The mathematical and computational analysis of how Parkinsonian damage occurs was intended to reveal a generic principle of neurochemical switching. The aim of this article is to test this principle in the specific case of animals exposed to a known PD risk factor. We monitored the temporal variations in nitric oxide (NO) within the brains of freely moving rats under stress from paraquat (PQ) injections. We then used temporal variations in NO levels to infer the presence of switching in oxidative stress levels. To capture the predicted switching process we made continuous measurements over a period of several days using NO sensors implanted in the brains of freely moving rats. Over the measurement period, animal groups were subjected to either (i) single administrations of PQ of various levels, or (ii) repeated administrations of a PQ dose of a fixed level. We believe the results of these experiments are: (i) supportive of a neurochemical switching mechanism in the initiation of self-sustaining Parkinsonian damage, and (ii) a demonstration of the value of LIVE for in vivo neurochemical measurement of previously unobservable temporal changes during neurodegeneration.

\section{MATERIALS AND METHODS}

\section{The use of a $P Q$ animal model}

We used a toxin based animal model that was known to us and that allowed PD risks to be introduced in a quantitatively and temporally deterministic manner. We selected the PQ animal model despite much debate regarding the ability of PQ to cross the blood brain barrier. Nonetheless, and because of its efficient redox cycling, PQ is widely used in animal models of PD. In particular, a strong correlation has been found between the vulnerability to PD damage and the level of PQ exposure [11-15]. For this reason, $\mathrm{PQ}$ was used to emulate the various levels of external toxic stress that form the inputs to the aforementioned theoretical model.

\section{The use of nitric oxide measurements as an indicator of oxidative damage under $P Q$ exposure}

We have recently developed and extensively characterised a Nafion ${ }^{\circledR}$-modified Pt sensor capable of real-time monitoring of brain extracellular $\mathrm{NO}$ in multiple brain regions [7-9]. In particular we have established a reliable method of directly measuring endogenously generated NO in tissues with the necessary sensitivity, spatial and temporal precision required 
for such a ubiquitous short-lived molecule. The technique requires the implantation of a Nafion ${ }^{\circledR}$-modified Pt sensor into a particular brain region, such that (through application of a suitable potential profile) it is possible to measure changes in concentration of $\mathrm{NO}$ on a timescale from milliseconds to days. Until recently the role of NO in the pathophysiology of complex brain disorders such as schizophrenia, Alzheimer's disease and PD has been hypothesized by indirect methods of analysis. However, the Nafion ${ }^{\circledR}$-modified Pt NO sensor possesses the necessary characteristics and precision for specific applications in animal models of disease [9], by providing direct, real-time, continuous monitoring of neurochemical levels.

For example, a recent application of the NO sensor in an animal model of schizophrenia has provided the first explicit biochemical evidence for the involvement of NO in the effects of the psychotomimetic drug phencyclidine hydrochloride (PCP) [10]. To the best of our knowledge the experiments described in this paper are the first in which continuous chronic realtime measurement of individual neurochemicals has been applied in vivo to the study of neurodegenerative conditions such as PD.

For our experiments we used in vivo measurement of NO to infer corresponding changes in oxidative stress levels. As a justification of this method, the literature on oxidative damage reveals that NO is closely related to oxidative damage in several ways. First, NO spontaneously reacts with another free radical, superoxide (SO) under diffusion limiting conditions to form peroxynitrite $\left(\mathrm{ONOO}^{-}\right)$; this is highly damaging to proteins and cell membranes and must be neutralized by the anti-oxidative pathways. During homeostasis SO is rapidly removed by high concentrations of scavenging enzymes called superoxide dismutases (SOD) with distinct isoenzymes located in the mitochondria, cytoplasm, and extracellular compartments [16, 17]. Under exposure to $\mathrm{PQ}$, the picture becomes more complex. Firstly, $\mathrm{PQ}^{+}$is re-oxidised back to $\mathrm{PQ}^{2+}$ by losing an electron to $\mathrm{O}_{2}$, resulting in the formation of the latter's reduced form $\mathrm{O}_{2}{ }^{-}$or $\mathrm{SO}$. PQ will also decouple the neuronal (and also potentially, endothelial) NO synthase (nNOS and eNOS) enzymes, thus reducing NO synthesis and producing SO instead. Both these mechanisms would intuitively lead to a reduction in NO concentration. However, PQ also elicits an inflammatory response through the NF- $\kappa$ B pathway, which leads to the expression of the inducible NO synthase (iNOS) that would compensate for the lower NO levels or even cause excess NO accumulation, which is also reported for other PD inducing toxins [18-20].
The NO sensors were implanted in the ventral striatum of a cohort of rats. After recovery from the surgery the rats were placed in cages where they could move freely and left until they exhibited normal behaviour. They were then subjected to subcutaneous (in the back of the subject) injections of various levels of PQ. The high sensitivity and selectivity of the NO sensors [7-9], in combination with continuous recording over several days ensured that long-term dynamic changes of NO in the rats were monitored in detail.

\section{NO monitoring in the ventral striatum}

Cell death in the substantia nigra (SN) and the associated motor dysfunction is frequently used as the defining characteristic of PD and on this basis the SN would seem a preferred region for sensor implantation. However, our experimental objective is to detect the initiation of Parkinsonian damage generally and not the appearance of the defining symptom of PD. Specifically, Braak's staging model and post-mortem evidence of widespread Parkinsonian damage in the brains of people with PD [21, 22], show that Parkinsonian damage is not localised to the $\mathrm{SN}$. As a result, sensors can be implanted in any brain area feasibly affected during staging and where measurements can be made with a high level of confidence. In this context, an extensive characterisation study of the NO sensor performance has already been made with it implanted in the ventral striatum [8]. This study provided the team with a reliable and reproducible protocol for implantation and validation of the sensor's performance in this brain area. Thus we measure NO in the ventral striatum, on the basis that: (i) we can do this reliably and reproducibly, and (ii) we are looking for the onset of Parkinsonian damage in this area - not the defining manifestation of motor dysfunction associated with SN cell death.

\section{Specificity of the sensed NO levels}

The NO signal displayed in all figures is an average level of extracellular NO measured around the modified sensor surface. This corresponds to interpreting the mathematical model as representing an extracellular compartment containing the neurons in the region of the ventral striatum covered by the sensors.

\section{NO sensor preparation and surgical implantation}

The microsensor is a Nafion ${ }^{\circledR}$-modified Pt disk electrode that has been extensively characterised both in 
vitro $[9,23]$ and in vivo $[7,8]$ and sold commercially by BlueBox Sensors Ltd., Dublin, Ireland. NO sensors were implanted following a previously described procedure. Coordinates for the ventral striatum with the skull levelled between bregma and lambda, were: $\mathrm{A} / \mathrm{P}+1.85, \mathrm{M} / \mathrm{L} \pm 1.3$ from bregma and $\mathrm{D} / \mathrm{V}-6.8$ from dura respectively. A reference electrode (8T Ag wires, $200 \mu \mathrm{m}$ bare diameter) was placed in the cortex and an auxillary electrode (8T Ag wires, $200 \mu \mathrm{m}$ bare diameter) attached to one of the support screws. The reference potential provided by the bare $\mathrm{Ag}$ wire in brain tissue is very similar to that of the saturated calomel electrode (SCE) used in the in vitro characterization [24]. The electrodes and probe were fixed to the skull with dental screws and dental acrylate (Associated Dental Products, Swindon, UK). The rats were anesthetized with the volatile anaesthesia Isoflurane, placed in a Kopf stereotaxic instrument and kept on a heating pad to prevent hypothermia. A $1 \mathrm{mLkg}^{-1}$ injection of the opioid analgesic buprenorphine is administered subcutaneously 10 minutes before the end of the surgery and the animal allowed to rest. The animal is monitored for the next few hours, before being transferred to a holding bowl where it remains for the duration of the experiment. The animal is allowed to recover for at least 24 hours prior to connection to the potentiostat. The desired potential $(+900 \mathrm{mV} v s$. Ag wire) is then applied to the NO sensor and the current is allowed to stabilise for approximately $4-5$ days to allow for baseline recordings. Following this period of stabilisation, systemic administrations of PQ were commenced.

\section{Mathematical model of $N O$ response to $P Q$}

The mathematical model predicting a systemic switching of oxidative stress $[4,5]$ is a generic illustration of ROS and $\alpha S$ interactions under abstract representations of all known PD risk factors. In addition to the theoretically predicted switching mechanism, the experimental responses to the PQ toxins have additional characteristics which are specific to this toxic risk. To help clarify the experimental investigation described here, the model in [5] was adapted for reactions specifically associated with exposure to PQ and for which there is significant supporting literature. The details of the adapted mathematical model are described in from the Section 3 of the Supplementary Material.

\section{Chemicals and solutions}

PQ (Methyl viologen dichloride hydrate) was purchased from Sigma Chemical Co. (Dublin, Ireland).
A $0.9 \%$ solution of saline was prepared by dissolving $0.9 \mathrm{~g} \mathrm{NaCl}$ in $100 \mathrm{~mL}$ doubly distilled water. All systemic administrations of PQ were made up in a solution of $0.9 \%$ saline and administered subcutaneously in a volume of $1 \mathrm{~mL}$.

\section{Animals}

Male Wistar rats (Charles River, U.K, 280-400 g) were housed, with a maximum of four per cage in a temperature $\left(17-23^{\circ} \mathrm{C}\right)$, humidity and light controlled (12 h light, $12 \mathrm{~h}$ dark cycle) environment. Food and water were available ad libitum. The animals were allowed to acclimatise for at least one week prior to surgery. All experimental procedures were performed under license in accordance with the European Communities Regulations 2002 (Irish Statutory Instrument 165/2013).

\section{Experimental protocols}

All experiments were carried out with each member of the animal group in its home bowl. Implanted electrodes were connected to the potentiostat through a six-pin Teflon ${ }^{\circledR}$ socket and free movement of the animal was allowed via a Raturn ${ }^{\circledR}$ system (Bioanalytical Systems, Inc, Indiana, USA).

\section{Single injection of $P Q$}

For low dose $\left(5 \mathrm{mgkg}^{-1}\right) \mathrm{PQ}$ administrations, two or four sensors were implanted bilaterally in the ventral striatum of 6 Wistar rats (total sensor $n=12$ ). Following 4-5 days of baseline recording the animal was subjected to a single $5 \mathrm{mgkg}^{-1}$ PQ injection and NO levels were recorded for a period of 72 hours (3 hours pre injection baseline and 69 hours post injection recording with time $=0$ denoting time of injection). For moderate dose $\left(30 \mathrm{mgkg}^{-1}\right) \mathrm{PQ}$ administrations, two or four sensors were implanted bilaterally in the ventral striatum of 5 Wistar rats (total sensor $n=13$ ). Following 4-5 days of baseline recording the animal was subjected to a single $30 \mathrm{mgkg}^{-1} \mathrm{PQ}$ injection) and NO levels were recorded for a period of 72 hours (3 hours pre injection baseline and 69 hours post injection recording with time $=0$ denoting time of injection).

\section{Repeated injection of $P Q$}

Significant responses in NO levels for single PQ doses only appeared in the larger $\left(30 \mathrm{mgkg}^{-1}\right)$ dose. For this reason we only performed experiments for repetitive exposures on this higher PQ dose. The exper- 
iment was performed with an animal group of 4 Wistar rats (total sensor $n=11$ ). Two or four sensors were implanted bilaterally in the ventral striatum. Following 4-5 days of baseline recording the animal was subjected to the first $30 \mathrm{mgkg}^{-1} \mathrm{PQ}$ injection and NO levels were recorded for a period of 72 hours (3 hours pre injection baseline and 69 hours post injection recording with time $=0$ denoting time of injection). After this 72 hour period the second $30 \mathrm{mgkg}^{-1} \mathrm{PQ}$ injection was administered and the NO levels recorded for another 69 hours with time $=72$ denoting time of injection. The final $30 \mathrm{mgkg}^{-1}$ administration was performed after this 138 hour recording period and the NO levels were recorded for another 69 hours with time $=141$ denoting time of injection. Thus in the four animal group NO levels were measured over 210 hour period. In one case it was possible to administer a fourth $30 \mathrm{mgkg}^{-1}$ injection after the 210 hour recording period, and this animal was studied for a further period. All injections were administered at $12 \mathrm{p} . \mathrm{m}$. on the particular day and at time $=0,72,141$ hour respectively. After termination of the experiments the rats were decapitated and the brains were removed and stored in $10 \%$ formaldehyde. The brains were transferred to $30 \%$ sucrose solution and stored for approximately 7 days at $4{ }^{\circ} \mathrm{C}$. The sensor placement was verified by sectioning the brains using a cryostat and an atlas of the rat brain for reference [25].

Previous work published by the group has illustrated that there is no difference in NO levels recorded from sensors implanted bilaterally in the ventral striatum, supporting further the choice of implantation protocol [8].

\section{Instrumentation and data presentation}

Constant potential amperometry was performed using previously described methods [7, 8]. All data presented had baselines normalised to $100 \%$ illustrating the overall change in current as a $\%$ of the pre-injection baseline level. This removes both inter electrode and inter animal variability by ensuring that the presented NO changes are representative of the data from all the animals used in each study. It is important to mention that the total baseline current consists of basal NO levels and a small contribution from the capacitance current formed from the double layer that exists between the NO sensor surface and the tissue matrix. In most cases the capacitance current is negligible compared to the faradaic response and can effectively be ignored, however, it is necessary to consider this when interpreting the results.

\section{RESULTS}

NO baseline measurement

Figure 1 summarises the results for the administration of single doses of PQ. Figure 1A shows the average baseline recording of NO levels in the ventral striatum using the Nafion ${ }^{\circledR}$-modified Pt sensor over a 90 hour period in the absence of any injection. It is evident that there is little deviation from the baseline level of $100 \%$ throughout the duration of sampling in any of the recordings. The slight fluctuations above and below basal levels are characteristic of circadian rhythms associated with the animals and validate previous assumptions made by other research groups [26]. The stability and repeatability of the baseline measurements confirm the suitability of the amperometric sensors for long-term recordings of NO levels in the living brain. Further validating plots are given in the Supplementary Material (Supplementary Figures 1 and 2).

\section{Effect of single $P Q$ injections}

We considered the effect on brain NO of two levels of dosage: $5 \mathrm{mgkg}^{-1}$ and $30 \mathrm{mgkg}^{-1}$ injections. These two concentrations were used as they represent a low and moderate dose of the toxin, and previous work has illustrated that both low and moderate doses have elicited contrasting behavioural affects in animals. In particular, Corasiniti et al. report behavioural changes following systemic injections of $20 \mathrm{mgkg}^{-1}$ and $100 \mathrm{mgkg}^{-1} \mathrm{PQ}$, including tremors, salivation and occasional wet dog shakes [27]. In the Corasiniti study, a $5 \mathrm{mgkg}^{-1}$ dose of the toxin failed to produce gross change in the subjects studied. Likewise, over the first 180 minutes our measurements of NO after $5 \mathrm{mgkg}^{-1}$ injection of the herbicide (Fig. 1B) showed no systemic change. This contrasts with previous investigations unrelated to the current PD study, but using the NO sensor, which displayed a response to various treatments and a return to baseline within this time frame [7-10]. To determine the long-term effect of PQ on NO levels, a total measurement period of 72 hours was chosen (3 hours pre injection baseline and 69 hours post injection recording with time $=0$ denoting time of injection). This period was deemed to be sufficient for any systemic drug affect to be recorded and corresponds to the time frames for change that were predicted in the mathematical model of systemic switching to damagingly high levels of endogenous ROS generation $[4,5]$. 

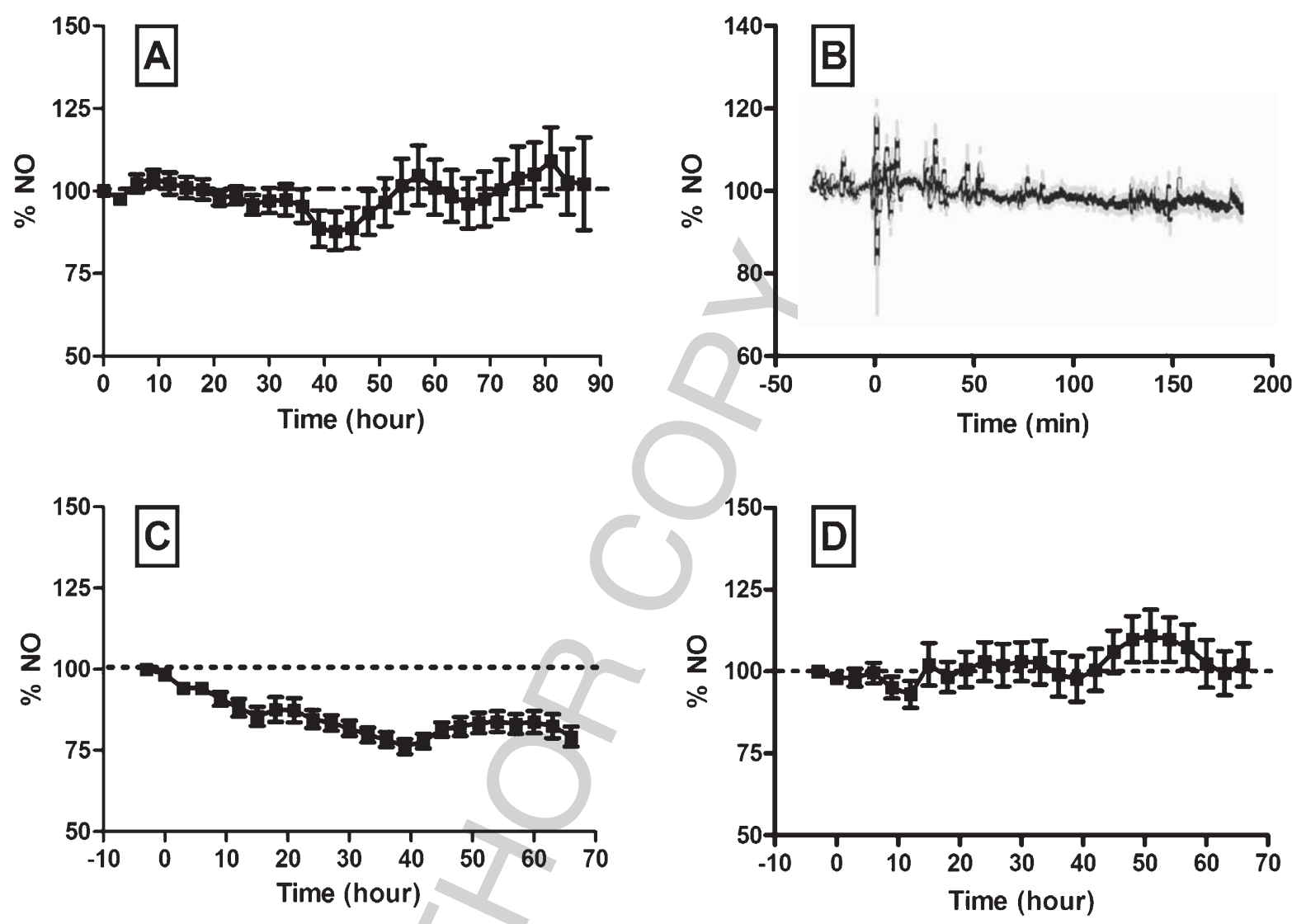

Fig. 1. Baseline and responses to single PQ injections. (A) Average 3 hour time intervals of baseline data recorded from amperometric NO sensors $(n=12)$ implanted in the ventral striatum of freely moving rats. Data represented as normalised current \pm S.E.M. (B) Average 3 hour response following $5 \mathrm{mgkg}^{-1}$ injections of PQ $(n=9)$. Grey lines represent S.E.M. (C) Average of all NO responses to $30 \mathrm{mgkg}^{-1}$ injections of PQ in freely moving animals $(n=13) .3$ hours pre injection baseline and 69 hours post injection recording with time $=0$ denoting time of injection (D) Average of all NO responses to $5 \mathrm{mgkg}^{-1}$ injections of PQ in freely moving animals $(n=12) .3$ hours pre injection baseline and 69 hours post injection recording with time $=0$ denoting time of injection.

\section{Effect of a single $5 \mathrm{mgkg}^{-1}$ injection of $P Q$}

Figure 1D shows the average response from NO sensors $(n=12)$ in an animal group over a 72 hour period following exposure to $5 \mathrm{mgkg}^{-1}$ of PQ. There are fluctuations above and below the baseline but the overall trend is one of minimal deviation from baseline, indicating that the animal's regulatory mechanisms are able to resist this level of toxic exposure. Having said this, it is important to mention that there were significant differences in the response of the 6 animals in this particular treatment group (see Supplementary Figure 2). The sensors implanted in three of the animals displayed transient decreases in current over the period monitored in contrast to the increases observed from the sensors implanted in the other three animals. Our sensor manufacturing, testing and calibration procedures are such that we are confident that the measured differences between animals are not an artefact of the sensor but are true reflections of differences in the animal responses. In this context, we believe the difference between responses reflects differences in animal metabolism and the corresponding different susceptibility to relatively low levels of toxic exposure.

\section{Effect of a single $30 \mathrm{mgkg}^{-1}$ injection of $P Q$}

Figure 1C illustrates the effect of one $30 \mathrm{mgkg}^{-1}$ injection $(n=13)$ of PQ on NO levels in the brains of a rat cohort ( 3 hours pre injection baseline and 69 hours post injection recording with time $=0$ denoting time of injection). There is a clear trend in the NO response observed over the 72 hours with a distinct decrease in NO levels recorded. There was a more noticeable change in the animal's behaviour in comparison to the 

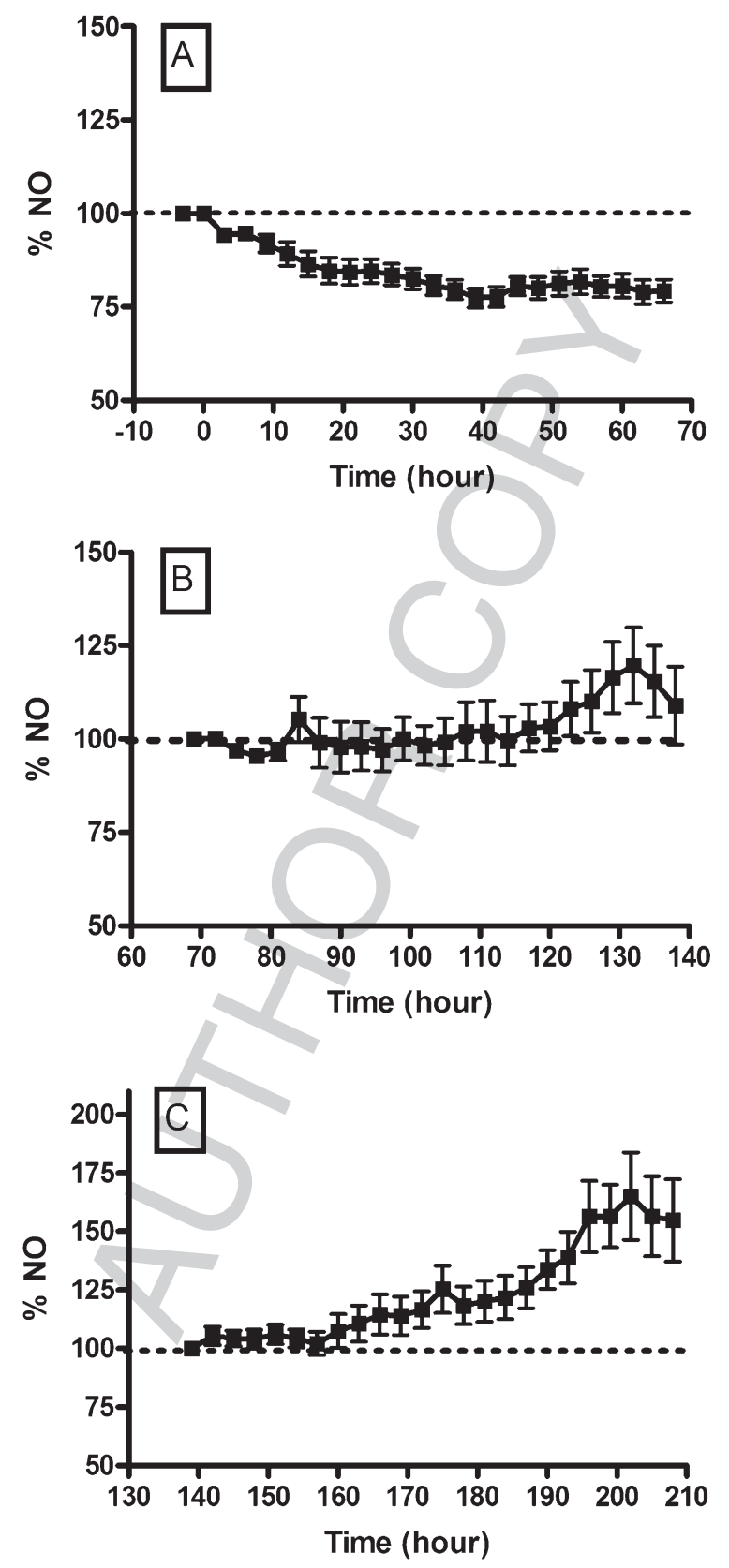

Fig. 2. The effect of multiple paraquat $\left(30 \mathrm{mg} . \mathrm{kg}^{-1}\right)$ injections on NO monitored in the ventral striatum of freely moving rats. (A) Average effect of first injection (0 hour, $n=11$ ), (B) second injection (72 hour, $n=11$ ), and (C) third injection (141 hour, $n=11$ ). Data represented as normalised current \pm S.E.M.

lower $5 \mathrm{mgkg}^{-1}$ dose including slight tremors and continual staring over the course of the recording. This is in line with other groups who report similar behavioural changes following administration of the toxin [28-31], and confirm that there is a dose related effect of the herbicide on NO levels. As described elsewhere, two major phenomena are most probably involved here: (i)-the decoupling of nNOS, switching to diaphorase activity and production of SO instead of NO; and (ii)a delayed increase in $\mathrm{SO}$, leading to a proportional increase in the NO-SO reaction, thus removing NO. eNOS is not explicitly considered here, but may play a 
role, even though its concentration is lower than $\mathrm{nNOS}$ in the brain tissue.

\section{Repeated exposure to $30 \mathrm{mgkg}^{-1} \mathrm{PQ}$ injections}

Figure 2 shows the effect of repeated injections of $30 \mathrm{mgkg}^{-1}$ PQ on the NO levels recorded using the amperometric sensors $(n=11)$. These are shown as snapshots of three time intervals during the 210 hour experiment described in Experimental Protocols. Figure $2 \mathrm{~A}$ covers the first 72 hours after the first injection at time $=0$; Fig. $2 \mathrm{~B}$ is a second 72 hour period after the second injection at time 72 hours; and Fig. 2C is a third 72 hour period after the third injection at time 141 hours. In all figures there is an averaged preinjection baseline period of 3 hours included; this is used to give the relative $100 \%$ level for each particular figure.

Figure 2A illustrates the effect of an initial exposure to the toxin over a 72 hour period. As previously observed for the single $30 \mathrm{mgkg}^{-1}$ injection, there is a systematic decrease in NO levels recorded over this period. Figure 2B shows the effect of the second $30 \mathrm{mg} / \mathrm{kg}^{-1}$ injection at time $=72$ hours. In this time interval, the decrease in NO relents and the NO levels remain close to baseline throughout the initial 50 hours following injection, after which a delayed increase is observed from baseline. This suggests that the second toxic exposure causes a secondary mechanism of action to take control and initiate an increase in NO levels. It is our understanding that this increase in NO levels may occur via a glutamatergic pathway and evidence exists supporting a mechanism by which PQ induces glutamate efflux [11, 12]. However, a definitive pathway has yet to be elucidated. It is well known that excitotoxicity is a process induced by $N$-methyl-Daspartate (NMDA) receptor activation through an NO mediated process. For example, Dugan and Choi have reported that excessive NMDA receptor activation is a central mechanism of neurodegeneration in several neurological diseases [32].

Figure $2 \mathrm{C}$ shows that, following a third exposure to $30 \mathrm{mgkg}^{-1}$, a clear increase in NO levels occurs over the subsequent 69-hour recording period for animals in the group. This experimental finding supports the theory that above a certain threshold of PQ exposure the diaphorase activity of NOS is superseded by toxin induced increase in glutamate efflux. Excessive activation of the NMDA receptor results in $\mathrm{Ca}^{2+}$ influx into the cell that is a prerequisite (along with the protein calmodulin) for the production of neuronal NO. However, it is important to emphasise that PQ also elicits an inflammatory response through the NF- $\kappa$ B pathway, which leads to the expression of the inducible NO synthase (iNOS) and to further NO production [18-20]. The NO then diffuses out from the cell into the extracellular space where it is detected by the amperometric sensor. Whatever the mechanism at work, it is clear that repetitive injections of the toxin alter production of NO. It is hypothesised that the increased NO produced diffuses across to dopaminergic terminals where it carries out a role in excitotoxicity, probably through its high affinity for the SO radical, resulting in the formation of the highly toxic anion $\mathrm{ONOO}^{-}$which is the key culprit of nitric oxide mediated toxicity. $\mathrm{ONOO}^{-}$is a lipid-permeable ion that has a wider range of chemical targets than NO. It can oxidise proteins, lipids, RNA and DNA. It inhibits enzyme function in the mitochondrial respiratory chain and decreases ATP synthesis resulting in a weakened energy metabolism of neurons [12].

Figure 2 provides us with a set of 'snapshots' of the effect of the three successive injections on the animal group over three successive time intervals. Each snapshot shows a different phase in the NO response as PQ injections are repeated, and illustrate the different neurochemical reactions that come into play at each stage, with the predicted shift to a consistently high level of NO occurring in the last stage after injection 3 (Fig. 2C). For completeness, a plot of the variations in $\mathrm{NO}$ over the entire experimental period is given in Fig 3. This plot of NO variation over the entire time period clearly shows that there is a systematic move to an elevated level of NO associated with the third injection (injection times are indicated by arrows). After this point the NO for all animals in the group increased in a characteristic manner to a high level of NO and stabilised at that value. The experimentally observed switching in NO levels in Fig. 3 should be compared with the simulations of the theoretically predicted bistable switching mechanism in $[4,5]$ and characterised by the feedback motif in [4].

The group experiment with $30 \mathrm{mgkg}^{-1}$ was terminated after 210 hours. However, we were able to monitor one unusually resilient member of the group over a longer period (13 days) that included a fourth $30 \mathrm{mgkg}^{-1}$ injection. It is unusual to obtain continuous neurochemical measurements for a subject over such a long period after sustained toxin insults. For this reason, the single animal results are included in the Supplementary Material, together with a mathematical model and simulations that relate specifically to the response characteristics displayed by this single subject. 


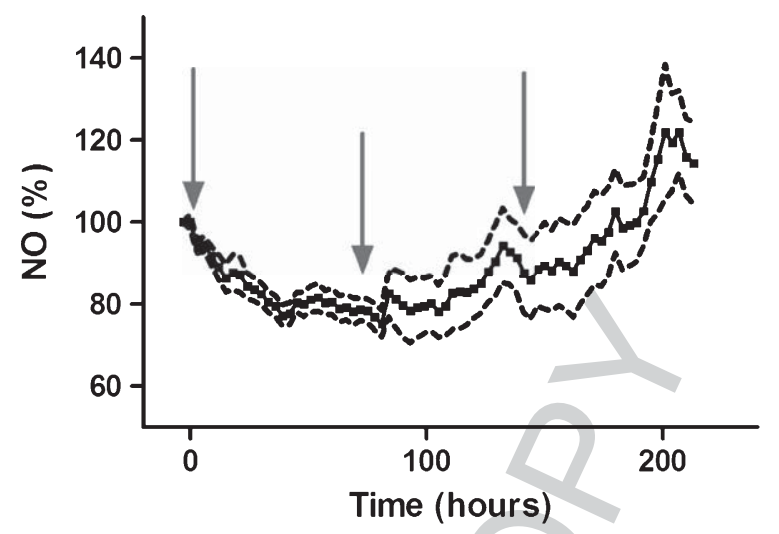

Fig. 3. Showing the variations in NO over the entire experimental period for an animal group exposed to three successive $30 \mathrm{mgkg}^{-1}$ injections of PQ at 72 hour periods. The experiment was terminated before the fourth injection.

\section{DISCUSSION}

Systematic changes in NO levels after a single $30 \mathrm{mgkg}^{-1} \mathrm{PQ}$ injection

With reference to Figs. $1 \mathrm{C}$ and $2 \mathrm{~A}$, we associate the initial dip in the sensed $\mathrm{NO}$ as an indicator of diaphorase action triggered by PQ. In this context, a number of recent publications have considered the importance of diaphorase activity in PQ-mediated toxicity [31, 33]. Cellular diaphorases are a class of enzymes that transfer electrons from NADPH to small molecules such as PQ [12, 34, 35]. Evidence suggests that NOS can act as a PQ diaphorase that causes uncoupling of the enzyme at the reductase domain, resulting in the generation of SO and depleting NO. Our findings with single $30 \mathrm{mgkg}^{-1}$ injections of PQ support this diaphorase hypothesis. It is noteworthy that in all animals a $30 \mathrm{mgkg}^{-1}$ injection produced a sustained decrease in NO levels in comparison to the apparent lack of response to a $5 \mathrm{mgkg}^{-1}$ injection. It is possible that at higher concentrations of PQ greater electron transfer occurs between the toxin and NADPH and this causes increased uncoupling of NOS and an overall increase in SO and decrease in NO. The NO current fails to recover back to pre-injection levels following the higher dose due to the efficient redox cycling of PQ that results in continuous uncoupling of NOS and inhibition of NO production.

\section{Systematic changes in NO levels after repeated $30 \mathrm{mgkg}^{-1} P Q$ injections}

The systematic changes shown in the animal groups (Figs. 2 and 3) after injection three provide supporting evidence of a predicted bistability-induced switching in neurochemical state within the rat brains. The bistability is one where excessive risk exposure causes endogenous oxidative stress to switch from a healthy homeostatic level to a damagingly high level of oxidative stress. The mathematical models in $[4,5]$ predict a switch like transition to damagingly high levels of ROS, and the animal group result in Fig. 2C (and for the entire time period in Fig. 3) is coherent with this prediction. Subsequent to the systemic switching further behaviours are to be expected that are associated with the severity and the type of the risk - we present models and simulations of the possible nature of these in the Supplementary Material.

\section{CONCLUSIONS}

Real-time measurements of NO in the ventral striatum of freely moving rats show the effect of PQ and its link to theoretically predicted changes in ROS. In particular the observation of a characteristic change in $\mathrm{NO}$ levels supports the theoretical prediction of a bistable neurochemical switching mechanism for exposure to PD risk factors above a certain magnitude. In the particular case of $\mathrm{PQ}$ injections, in vivo $\mathrm{NO}$ measurements in rats exhibit a switching phenomenon for repeated toxic insults of $30 \mathrm{mgkg}^{-1}$ while low levels $\left(5 \mathrm{mgkg}^{-1}\right)$ do not. There was variability between the responses of the animals used in the experimental groups, but we believe that this is indicative of intrinsic differences in susceptibility between animals in the group. The observation that permanent PD damage was initiated by repeated toxic insults, and that there were differences in susceptibility of the subjects may have relevance 


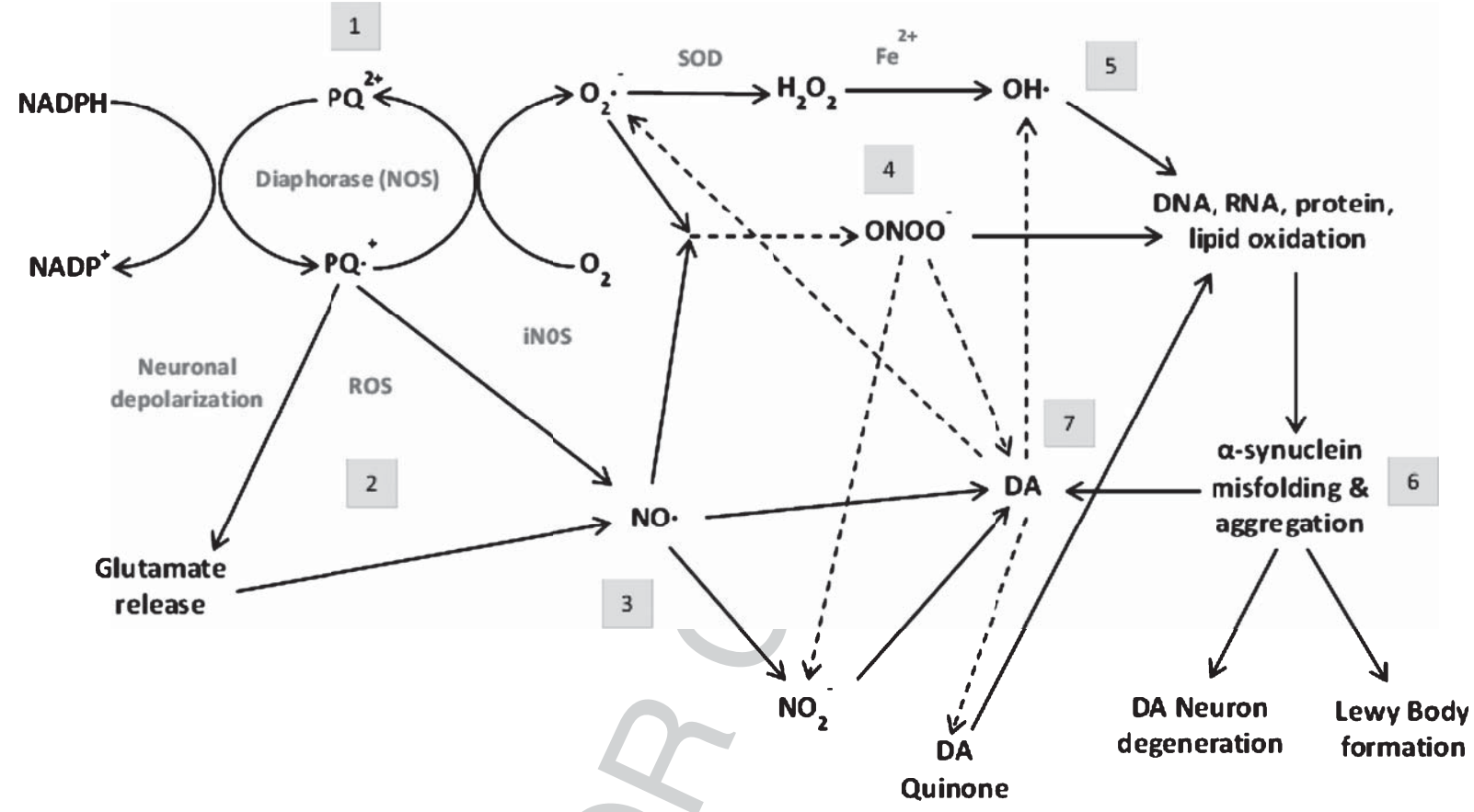

Fig. 4. Proposed mechanism for PQ induced toxicity (1) Diaphorase activity displayed by NOS in the presence of PQ. NOS transfer electrons from NADPH to PQ causing inhibition of complex I of the mitochondrial respiration chain (ETC) resulting in deficient ATP synthesis and neuronal vulnerability. PQ. ${ }^{+}$undergoes reoxidation in the presence of $\mathrm{O}_{2}$ generating $\mathrm{O}_{2} \cdot{ }^{-}$(SO) (2) Following repeated exposure PQ. ${ }^{+}$induces excessive glutamate release that results in hyperactivation of NMDA receptors causing an increase in NO production. An alternative source of increasing NO levels occurs via increased iNOS expression (3) Excess levels of NO react with $\mathrm{O}_{2} \cdot{ }^{-}$resulting in the formation of toxic ONOO ${ }^{-}$, $\mathrm{NO}$ also has a role in mediating DA release and oxidation through the action of its metabolite $\mathrm{NO}_{2}^{-}$(4) $\mathrm{ONOO}^{-}$produced following the spontaneous reaction of $\mathrm{NO}$ and $\mathrm{O}_{2}{ }^{-}$directly oxidises DA or indirectly via decomposition to $\mathrm{NO}_{2}{ }^{-}$(5) Oxidation of proteins, lipids, \& DNA by $\operatorname{ROS}\left(\mathrm{OH} \cdot \& \mathrm{ONOO}^{-}\right)$and disruption to homeostatic degradation of mis-folded proteins (6) $\alpha$-S mis-folding \& aggregation in dopaminergic neurons prevents DA storage in vesicles resulting in increased DA oxidation, $\alpha$-S mis-folding \& aggregation make up Lewy Bodies (7) DA not

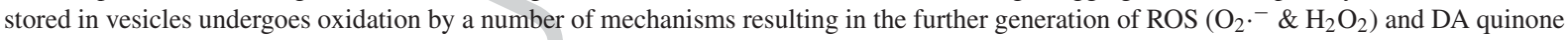
which all feedback into eventual $\alpha-S$ mis-folding \& aggregation.

in the debate concerning human exposure to certain agricultural and industrial chemicals.

We believe that the NO measurements described here support an important theoretical prediction concerning how self-sustaining Parkinsonian damages starts. Specifically the data provides experimental evidence of a bistable neurochemical switch that is activated by stresses from PD risk factors. The mathematical models that motivated our experiments were for a generalised situation and required changes to describe the specific risk presented by PQ. This point suggests that although each PD risk factor or combination of risk factors, maybe different, the core mechanism that initiates the disease state can be described by the unifying phenomenon of a bistable neurochemical system that causes a switch to elevated ROS levels. A further unifying observation is that all risk factors acting on the neurochemical switch mediate the neuronal ATP availability - supporting the role for neuronal energy deficits as a unifying feature of known PD risk factors [36-38].

A caveat is that the system that characterises the NO response to PQ (Fig. 4) is more complex than the generic mathematical model used to motivate our experiments [4]. Such differences are to be expected, as the original model was developed as a generic feedback motif that is prototypical of the transition between healthy and disease states. To answer this point we have included in the Supplementary Material a model that includes PQ specific reactions and captures the specific NO dynamics observed during the in vivo measurements for an individual animal's responses.

Other potentially useful findings for PD research include (i) the importance of both the size and timing of toxic exposure, (ii) the variations between responses to individual external stresses and, (iii) that the development of damaging levels of oxidative species seems to be intrinsically dynamic. This last point is relevant for 
experiments based upon point measurements at one specific time - usually the end point. Such observations will potentially show widely differing results, not because of genuine experimental differences, but because the measurement points occur at different phases during dynamic changes in neurochemical levels.

Beyond the specific results concerning PD, the experiments shown here demonstrate the value of LIVE for continuous long-term sensing of neurochemical concentrations as a tool for neurodegenerative disease research in general. For example, the temporal variations revealed in Figs. 2 and 3, would have been extremely difficult, if not impossible, to detect using any other sensing technology. The long-term variations in neurochemical levels illustrate the importance of continuous measurements as a tool for obtaining a global and comprehensive picture of change during neurodegeneration. In summary, the findings from this body of work lend support to the suitability of such sensors as an important tool in monitoring the manifestation of neurodegenerative disease where oxidative stress is a central feature. It would be of great interest to combine various other microsensors for real-time quantification of several important markers in PD. The BioAnalytics Laboratory at NUI Maynooth has a pipeline of such sensors that allow continuous realtime detection of markers such as glutamate, hydrogen peroxide and ascorbic acid. Collectively these sensors would allow unprecedented recordings in pre-clinical models of PD and illustrate for the first time the manifestation of this debilitating disease.

\section{ACKNOWLEDGMENTS}

The neurochemical sensor development and realtime neurochemical measurements described here was supported by Science Foundation Ireland (03/IN3/B376), Enterprise Ireland (PC/2008/004) and the Higher Education Authority under the Programme for Research in Third Level Institutions (PRTLI) Cycle 4. The team would also like to especially thank $\mathrm{Mr}$ Michael Kelly and Hilde-Ulrichs-Stiftung für Parkinsonforschung for their charitable donation to assist with the in vivo work. The mathematical modelling and simulation of neurochemical sensor data was supported by the Fonds de Recherche du Quebec - Nature et Technologies. The mathematical modelling and systems approach to Parkinson's disease was supported by Science Foundation Ireland Research Professor Award (03/RP1/I382).

\section{AUTHOR CONTRIBUTIONS}

N.J.F and S.L.O.R contributed equally in obtaining LIVE NO determinations. J.P.L provided insight into analysis and presentation of LIVE data. M.C. developed the mathematical model, performed all simulations comparing in vivo with in-silico performance. P.W. contributed to experimental design, and the writing and editing of the paper.

\section{CONFLICT OF INTEREST}

The authors have no conflict of interest to report.

\section{REFERENCES}

[1] deRijk MC, Tzourio C, Breteler MMB, Dartigues JF, Amaducci L, LopezPousa S, ManubensBertran JM, Alperovitch A, \& Rocca WA (1997) Prevalence of parkinsonism and Parkinson's disease in Europe: The europarkinson collaborative study. J Neurol Neurosurg Psychiatry, 62, 10-15.

[2] Lotharius J, \& Brundin P (2002) Pathogenesis of Parkinson's disease: Dopamine, vesicles and alpha-synuclein. Nat Rev Neurosci, 3, 932-942.

[3] Cassarino DS, \& Bennett JP (1999) An evaluation of the role of mitochondria in neurodegenerative diseases: Mitochondrial mutations and oxidative pathology, protective nuclear responses, and cell death in neurodegeneration. Brain Res Rev, 29, 1-25.

[4] Cloutier M, Middleton R, \& Wellstead P (2012) Feedback motif for the pathogenesis of Parkinson's disease. Iet Systems Biol, 6, 86-93.

[5] Cloutier M, \& Wellstead P (2012) Dynamic modelling of protein and oxidative metabolisms simulates the pathogenesis of Parkinson's disease. Iet Systems Biol, 6, 65-72.

[6] Tyson JJ, Chen KC, \& Novak B (2003) Sniffers, buzzers, toggles and blinkers: Dynamics of regulatory and signaling pathways in the cell. Current Opin Cell Biol, 15, 221-231.

[7] Finnerty NJ, O'Riordan SL, Brown FO, Serra PA, O'Neill RD, \& Lowry JP (2012) In vivo characterisation of a Nafion (R)modified Pt electrode for real-time nitric oxide monitoring in brain extracellular fluid. Anal Methods, 4, 550-557.

[8] Finnerty NJ, O'Riordan SL, Palsson E, \& Lowry JP (2012) Brain nitric oxide: Regional characterisation of a realtime microelectrochemical sensor. J Neurosci Methods, 209, 13-21

[9] Brown FO, Finnerty NJ, \& Lowry JP (2009) Nitric oxide monitoring in brain extracellular fluid: Characterisation of Nafion (R)-modified Pt electrodes in vitro and in vivo. Analyst, 134, 2012-2020.

[10] Palsson E, Finnerty N, Fejgin K, Klamer D, Wass C, Svensson L, \& Lowry J (2009) Increased cortical nitric oxide release after phencyclidine administration. Synapse, 63, 1083-1088.

[11] Shimizu K, Matsubara K, Ohtaki K, Fujimaru S, Saito O, \& Shiono H (2003) Paraquat induces long-lasting dopamine overflow through the excitotoxic pathway in the striatum of freely moving rats. Brain Res, 976, 243-252.

[12] Dinis-Oliveira RJ, Remiao F, Carmo H, Duarte JA, Navarro AS, Bastos ML, \& Carvalho F (2006) Paraquat exposure as an etiological factor of Parkinson's disease. Neurotoxicology, 27, 1110-1122. 
[13] McCormack AL, Atienza JG, Johnston LC, Andersen JK, Vu S, \& Di Monte DA (2005) Role of oxidative stress in paraquatinduced dopaminergic cell degeneration. J Neurochem, 93, 1030-1037.

[14] McCormack AL, Atienza JG, Langston JW, \& Di Monte DA (2006) Decreased susceptibility to oxidative stress underlies the resistance of specific dopaminergic cell populations to paraquat-induced degeneration. Neuroscience, 141, 929-937.

[15] McCormack AL, \& Di Monte DA (2003) Effects of L-dopa and other amino acids against paraquat-induced nigrostriatal degeneration. J Neurochem, 85, 82-86.

[16] Pacher P, Beckman JS, \& Liaudet L (2007) Nitric oxide and peroxynitrite in health and disease. Physiol Rev, 87, 315-424.

[17] Beckman JS, \& Koppenol WH (1996) Nitric oxide, superoxide, and peroxynitrite: The good, the bad, and the ugly. Am J Physiol, 271, C1424-C1437.

[18] He Y, Imam SZ, Dong ZJ, Jankovic J, Ali SF, Appel SH, \& Le WD (2003) Role of nitric oxide in rotenone-induced nigro-striatal injury. J Neurochem, 86, 1338-1345.

[19] Przedborski S, JacksonLewis V, Yokoyama R, Shibata T, Dawson VL, \& Dawson TM (1996) Role of neuronal nitric oxide in 1-methyl-4-phenyl-1,2,3,6-tetrahydropyridine (MPTP)-induced dopaminergic neurotoxicity. Proc Natl Acad Sci U S A, 93, 4565-4571.

[20] Shen T, Zhu Q-X, Yang S, Ding R, Ma T, Ye L-P, Wang L-J, Liang Z-Z, \& Zhang X-J (2007) Trichloroethylene induce nitric oxide production and nitric oxide synthase mRNA expression in cultured normal human epidermal keratinocytes. Toxicology, 239, 186-194.

[21] Braak H, \& Del Tradici K (2008) Nervous System Pathology in Sporadic Parkinsons Disease. Neurology, 70, 1916-1925.

[22] Przuntek H, \& Muller TPR (2004) Diagnostic staging of Parkinson's disease: Conceptual aspects. J Neural Transm, 111, 201-216.

[23] Brown FO, \& Lowry JP (2003) Microelectrochemical sensors for in vivo brain analysis: An investigation of procedures for modifying Pt electrodes using Nafion. Analyst, 128, 700-705.

[24] O’Neill RD, Lowry JP, \& Mas M (1998) Monitoring brain chemistry in vivo: Voltammetric techniques, sensors, and behavioral applications. Critical Rev Neurobiol, 12, 69-127.

[25] Paxinos G, \& Watson C (1998) The Rat Brain in Stereotaxic Coordinates. Academic Press.

[26] Heinzen EL, \& Pollack GM (2002) Use of an electrochemical nitric oxide sensor to detect neuronal nitric oxide production in conscious, unrestrained rats. J Pharmacol Toxicol Methods, 48, 139-146.

[27] Corasaniti MT, Strongoli MC, Rotiroti D, Bagetta G, \& Nistico G (1998) Paraquat: A useful tool for the in vivo study of mechanisms of neuronal cell death. Pharmacol Toxicol, 83, 1-7.

[28] Hara S, Mukai T, Kuriiwa F, Yanase T, Kurosaki K, Kano S, \& Endo T (2000) Suppression of paraquat-induced wet dog shakes by nitric oxide synthase inhibitors in rats. Life Sci, 66, PL189-PL194.

[29] Hara S, Mukai T, Kurosaki K, Kuriiwa F, Yanase T, Kano S, \& Endo T (2001) No parallel relationship between nitric oxide production and wet dog shakes susceptible to nitric oxide synthase inhibitors following systemic administration of paraquat in rats. Arch Toxicol, 74, 775-782.

[30] Xu XY, \& Miller KJ (1998) Prevention of DOI-mediated wet dog shakes by inhibitors of nitric oxide synthase. Brain Res, 804, 337-340.

[31] Moran JM, Ortiz-Ortiz MA, Ruiz-Mesa LM, \& Fuentes JM (2010) Nitric oxide in paraquat-mediated toxicity: A review. J Biochem Mol Toxicol, 24, 402-409.

[32] Dugan LL, \& Choi DW (1999) Hypoxic-ischemic brain injury and oxidative stress. In Basic Neurochemistry: Molecular, Cellular and Medical Aspects, 6th edition. Siegel GJ, Agranoff BW, Albers RW, Fisher SK, Uhler MD, eds, Lippincott-Raven, Philadelphia.

[33] Ortiz-Ortiz MA, Moran JM, Gonzalez-Polo RA, NisoSantano M, Soler G, Bravo-San Pedro JM, \& Fuentes JM (2009) Nitric oxide-mediated toxicity in paraquat-exposed SH-SY5Y cells: A protective role of 7-nitroindazole. $\mathrm{Neu}$ rotox Res, 16, 160-173.

[34] Day BJ, Patel M, Calavetta L, Chang LY, \& Stamler JS (1999) A mechanism of paraquat toxicity involving nitric oxide synthase. Proc Natl Acad Sci U S A, 96, 12760-12765.

[35] Dicker E, \& Cederbaum AI (1991) NADH-dependent generation of reactive oxygen species by microsomes in the presence of iron and redox cycling agents. Biochem Pharmacol, 42, 529-535.

[36] Matsuda W (2012) Imaging of dopaminergic neurons and the implications for Parkinson's disease In Systems Biology of Parkinson's Disease, Wellstead P, Cloutier M, eds, Springer, New York.

[37] Wellstead P, \& Cloutier M (2012) Modelling and simulation of brain energy metabolism: Energy and Parkinson's disease In Systems Biology of Parkinson's Disease, Wellstead P, Cloutier M, eds., Springer, New York.

[38] Bolam JP, \& Pissadaki EK (2012) Living on the edge with too many mouths to feed: Why dopamine neurons die. Mov Disord, 27, 1478-1483. 